\title{
Information du public sur les essais cliniques et la recherche
}

\author{
Yannick Plétan, ${ }^{1}$ Fä̈ez Zannad, ${ }^{2}$ Patrice Jaillon ${ }^{3}$ et les participants à la table ronde \\ $n^{\circ} 1$ de Giens XVIII ${ }^{+*}$ \\ 1 Pfizer, Paris, France \\ 2 CIC-INSERM-CHU, Nancy, France \\ 3 CHU Saint-Antoine, Paris, France
}

\section{Résumé}

\begin{abstract}
Que ce soit pour restaurer l'image ternie de la recherche clinique vis-à-vis du grand public ou pour développer de nouvelles méthodes de recrutement des volontaires sains ou des patients dans les essais cliniques, il existe un besoin de recommandations permettant in fine d'optimiser l'information sur la recherche. Alors que l'arsenal légal et réglementaire français serait plutôt libéral en la matière, il existe actuellement et sans justification réelle une réticence des promoteurs d'essais à recourir à la publicité pour les essais. Un groupe de chercheurs cliniciens universitaires publics et industriels a tenu une table ronde en présence de représentants des autorités de santé, de journalistes, de membres des Comités Consultatifs de Protection des Personnes dans la Recherche Biomédicale, de représentants de la Sécurité Sociale, d'associations de malades et de consommateurs et d'autres instances administratives françaises afin d'émettre un certain nombre de recommandations qui peuvent s'énoncer comme suit :

- il n’y a pas nécessité d'augmenter les contraintes légales ou réglementaires ;

- les promoteurs d'essais doivent être informés sur les possibilités de recourir à l'information directe du grand public sur les essais cliniques et en faire usage ;

- une « charte professionnelle de bonne pratique » de communication sur les essais devrait être développée ;

- toute la chaîne des professionnels devrait être incluse dans la plate-forme de communication ;

- l'information de proximité du patient devrait être privilégiée (médecin traitant et presse régionale) ;

- le développement de répertoires et de sites internet dédiés devrait être encouragé, qu'ils soient accessibles non seulement aux professionnels mais aussi aux patients et aux non-professionnels ;

- une vraie pédagogie pour les médecins, personnels de santé non-professionnels de l'essai clinique, devrait être mise en place ;

- les médias devraient être formés aux bases de la recherche clinique.
\end{abstract}

Mots clés : information, public, essais cliniques, recherche

Texte reçu le 6 mai 2003 ; accepté le 23 mai 2003

\section{Introduction}

Au moment même où la table ronde consacrée à ce sujet se réunissait à Giens, un magazine "santé grand public » titrait en première de couverture, pour partie en caractères rouges : « 400000 Français louent leur corps à la médecine - Qui teste nos médicaments ? - Cobayes humains : sélection, rémunération, risques. » ${ }^{[1]}$
Voici en quelques mots résumée la perception qu'ont certains médias, et par suite le public de façon plus générale, de la recherche clinique en France en 2002. Au même moment, un échantillon de ces chercheurs cliniciens français, de leurs autorités tutélaires, et un aréopage de représentants des comités assurant la protection desdits sujets se réunissaient en se demandant comment, en particulier, la presse spécialisée et grand

$\dagger$ Pour la liste des participants, voir en fin d'article.

* Les articles, analyses et propositions issus des Rencontres de Pharmacologie Clinique de Giens n'engagent que leurs auteurs et ne préjugent pas de la position de leur organisme de tutelle. 
public, et les autres médias pourraient se conjuguer pour favoriser le développement de la recherche clinique sur notre territoire national, un sujet récurrent de préoccupation, ${ }^{[2]}$ tout en aidant activement au recrutement de patients dans nos essais cliniques de médicaments. Un échantillon de ce même groupe s'était entendu dire quelques semaines plus tôt lors d'une réunion sur ce thème au Sénat que la notion même de « cobayes humains » appartenait désormais, et c'était heureux ainsi, au passé.

Ce même thème avait fait l'objet de la $10^{\text {ème }}$ Journée de l'Association pour le Développement de la Pharmacologie Clinique à Paris en Octobre 1997. ${ }^{[3]}$

\section{Position du problème : délimitation du sujet}

Le sujet, par définition très large, a été volontairement circonscrit de la façon suivante :

- par «public », on comprendra les patients participant ou susceptibles de participer à des essais cliniques, leur entourage, et par extension toute personne concernée par le sujet ;

- par « recherche biomédicale », les essais cliniques de médicaments ;

- avec « information », on considèrera aussi les initiatives de « formation » s'adressant aux mêmes cibles ;

- par « support», tous les supports potentiels (presse tous médias, web, affichage, documents spécifiques) ;

- la question particulière de l'information en vue du recueil du consentement a été considérée hors sujet ;

- en revanche, l'information du sujet sur les résultats globaux de la recherche, telle qu'édictée par la loi « Droit des Malades » du 4 mars 2002, ${ }^{[4]}$ sera discutée.

\section{Cadre réglementaire et position des instances de contrôle}

Une perception traditionnelle parmi les promoteurs d'essais cliniques est que la communication en direction du grand public à propos des essais cliniques est fortement réglementée. Cette perception peut résulter des interdictions faites par la loi aux industriels du médicament de communiquer sur leurs produits en direction du grand public. ${ }^{[5]}$ Elle peut aussi provenir du constat que de nombreuses instances existent, qui organisent la recherche biomédicale pour s'assurer de sa conduite pertinente et éthique. Mais en définitive, dans notre pays, il n'y a pas de cadre légal ni réglementaire spécifique concernant les campagnes de recrutement des patients par voie d'affiche, de presse, par la radio ou sur internet.

La Directive Européenne sur les Essais Cliniques, ${ }^{[6]}$ dont la transposition en droit national est prévue pour mai 2003, demande, à l'article 6-k, que les modalités de recrutement soient portées à la connaissance d'un Comité d'Ethique, soit en France à un Comité Consultatif de Protection des Personnes dans la Recherche Biomédicale (CCPPRB).

Les informations qui suivent résultent pour l'essentiel d'expériences individuelles de promoteurs qui sont citées plus loin dans le texte et en référence.

Un avis non public du Comité Consultatif National d'Ethique (CCNE) d'avril 2001 (CCNE, données non publiées), en réponse à une question écrite, insiste sur l'éthique du message utilisé, son caractère simple, factuel, portant sur les bénéfices et les risques associés à la recherche ainsi que sur ses modalités, exception faite de la mention de l'indemnisation éventuelle. Le CCNE rappelle également les rôles du CCPPRB et de la Commission Nationale de l'Informatique et des Libertés (CNIL) en matière d'essais cliniques.

De même, un avis du Conseil National de l'Ordre des Médecins (CNOM) de décembre 2001 (Laboratoire 3M, données non publiées) fait une différence entre essais sans bénéfice individuel direct (SBID) (promotion plutôt permise) et avec BID (plus restrictif, devant impliquer le médecin traitant), et insiste sur l'importance qu'a l'investigateur de tenir le médecin traitant informé de l'essai dans lequel son patient est impliqué.

L'Afssaps (Agence française de sécurité sanitaire des produits de santé), consultée à ce sujet, considère qu'il n'appartient pas à la Commission de la Publicité de se prononcer sur les essais cliniques et qu'il ne relève pas non plus de l'Unité Essais Cliniques, d'une manière générale, de se prononcer systématiquement sur les procédures de recrutement dans les essais. Cette dernière pourrait en revanche répondre à toute saisine qui lui serait faite à ce sujet.

A l'instar de la France, les autres pays européens n'ont pas de cadre légal pour l'information concernant les essais cliniques. Il existe encore assez peu d'usage en la matière sinon dans les pays scandinaves et aux Pays-Bas où la pratique se répand. Cependant, le 3 octobre 2002, la Commission Environnement du Parlement Européen a voté à la majorité contre le projet de la Commission Européenne de communication vers le grand public sur certains médicaments (asthme, SIDA, diabète) pour une période test de 5 ans. Bien que sans portée directe sur notre démarche, ce vote indique les limites que les instances européennes voient en matière d'information.

Aux Etats-Unis, la communication sur les essais est libre sous réserve d'approbation par les «Institutional Review Boards (IRB) », selon le principe que l'information sur l'essai est autorisée dès lors que l'essai lui-même l'est. Ce principe de transparence est en ligne avec la section $113 \mathrm{du}$ « Modernization Act » de la «US Food and Drug Administration » (US FDA), ${ }^{[7]}$ lui- 
même repris par PhRMA (Pharmaceutical Research and Manufacturers of America), représentant l'industrie pharmaceutique américaine. ${ }^{[8]}$ Un site internet de la US FDA est destiné à mettre cette information à la disposition du public, sauf opposition expresse et justifiée par le promoteur.

Au Japon, depuis juin 1999, des règles voisines s'exercent, mais le nom du produit ne doit pas être mentionné, ce qui n'empêche nullement des campagnes massives en faveur des essais cliniques et un recrutement actif, en particulier par le canal des sites internet.

\section{Recommandations de la table ronde concernant le cadre réglementaire}

L'ensemble des participants, dont chacun représente cependant un angle d'observation différent, est d'accord sur une opposition de principe au développement d'une législation spécifique.

Alors qu'il existe un consensus sur le principe que les modalités d'information qui seront utilisées soient soumises avec le dossier global présenté pour avis au CCPPRB, il n'a pas été jugé nécessaire de solliciter un avis préalable et spécifique du CCPPRB sur les documents d'information eux-mêmes.

Pour rappel, la directive essais cliniques - actuellement en cours de rédaction - et relative au dossier à déposer au CCPPRB, prévoit le dépôt des procédures de recrutement et des documents utilisés pour cela.

A noter également que certaines remarques et propositions ont été émises par le groupe telles que :

- réticence à une distinction entre essais avec et sans BID (caractère discriminatoire et opposition trop caricaturale du risque/bénéfice entre les deux modalités);

- proposition d'établir une "charte de bon usage » (déontologie, crédibilité, intelligibilité, absence d'assimilation possible à une publicité pour un médicament ou une firme, modalités d'utilisation de centres d'appel).

Un point toutefois est resté en suspens : faut-il mentionner (au nom de la transparence) ou taire (pour éviter le caractère mercantile) le niveau de l'indemnisation des patients ?

\section{Quelle est la demande en matière d'information?}

Cette question est centrale à la compréhension du problème et préalable à l'émission de recommandations. Pour cerner la réponse à cette question, une approche par enquête a été utilisée.

Diverses enquêtes ont été utilisées :

- enquête d'opinion réalisée auprès d'un petit échantillon de consommateurs ;
- enquête auprès des patients appartenant à la clientèle de généralistes investigateurs (tableau I) ;

- enquête auprès de médecins généralistes réalisant des essais cliniques (tableau II) ;

- enquête auprès d'infirmières dans deux CHU (tableau III) ;

- enquête auprès de parents d'enfants vus à l'hôpital.

Les résultats qui en ressortent sont :

1. Les essais chez l'homme sont perçus comme une nécessité (90\% des patients [enquête réalisée auprès de la patientèle de médecins investigateurs ; Giacomino A, données non publiées]), y participer est une bonne action (73\% du grand public), ${ }^{[9]}$ et ceux réalisés dans le cadre de la médecine libérale sont

Tableau I. Opinion de patients concernant les essais thérapeutiques. Questionnaires remis au hasard dans la salle d'attente de médecins investigateurs (78 réponses exploitables) ${ }^{\text {a }}$

\begin{tabular}{|c|c|}
\hline Question & Réponses positives \\
\hline $\begin{array}{l}\text { 1. Avez-vous déjà participé à une recherche pour } \\
\text { un essai thérapeutique? }\end{array}$ & $12,8 \%$ \\
\hline 2. Avez-vous été satisfait de votre participation? & $11,1 \%$ \\
\hline 3. Vous a-t-on déjà proposé de participer? & $14,1 \%$ \\
\hline $\begin{array}{l}\text { 4. Si votre médecin vous proposait de participer, } \\
\text { seriez-vous plutôt d'accord? }\end{array}$ & $72,0 \%$ \\
\hline \multicolumn{2}{|l|}{$\begin{array}{l}\text { 5. Une personne de votre entourage proche } \\
\text { a-t-elle participé à un essai clinique de } \\
\text { médicament? }\end{array}$} \\
\hline oui & $11,8 \%$ \\
\hline non & $68,0 \%$ \\
\hline ne sait pas & $19,7 \%$ \\
\hline \multicolumn{2}{|l|}{$\begin{array}{l}\text { 6. Considérez-vous que l'information que vous } \\
\text { avez sur les essais cliniques est : }\end{array}$} \\
\hline bonne & $19,2 \%$ \\
\hline moyenne & $23,0 \%$ \\
\hline pas bonne & $57,7 \%$ \\
\hline \multicolumn{2}{|l|}{$\begin{array}{l}\text { 7. Considérez-vous que les essais cliniques et } \\
\text { thérapeutiques réalisés chez l'homme volontaire } \\
\text { sont nécessaires? }\end{array}$} \\
\hline oui & $89,7 \%$ \\
\hline non & $0,0 \%$ \\
\hline sans réponse & $11,3 \%$ \\
\hline $\begin{array}{l}\text { 8. Désirez-vous avoir plus d'informations sur les } \\
\text { essais cliniques? }\end{array}$ & $85,7 \%$ \\
\hline \multicolumn{2}{|l|}{$\begin{array}{l}\text { 9. Par quel moyen ? (par ordre de priorité } \\
\text { décroissante): }\end{array}$} \\
\hline & 1. Médecin \\
\hline & 2. Journaux \\
\hline & $\begin{array}{l}\text { 3. TV (ex-aequo } \\
\text { avec 2) }\end{array}$ \\
\hline & 4. Radio \\
\hline & 5. Internet \\
\hline
\end{tabular}

a Document complémentaire avec commentaires patients et analyse. Avec autorisation. Enquête réalisée auprès de la patientèle de médecins investigateurs ; Giacomino A, données non publiées. 
Tableau II. Opinion de médecins dits « investigateurs ». Questionnaire envoyé par e-mail à différents groupes de médecins (investigateurs, FMC, forum informatique) [26 réponses spontanées exploitables] ${ }^{\mathrm{a}}$

\begin{tabular}{|c|c|c|}
\hline Question & Réponse affirmative & Réponse négative ou absence \\
\hline 1. Faites-vous des essais cliniques? & 23 & 3 \\
\hline 2. Avez-vous eu des propositions d'essais? & 25 & 1 \\
\hline 3. Avez-vous envie de faire des essais ? & 21 & 5 \\
\hline 4. Etes-vous satisfait de votre participation aux essais cliniques? & 21 & 2 \\
\hline 5. Vos patients ont-ils été satisfaits de participer? & \multicolumn{2}{|c|}{ Les 23 médecins ont répondu « oui » } \\
\hline \multicolumn{3}{|l|}{ 6. Votre information sur les essais cliniques est-elle : } \\
\hline mauvaise & 16 & \\
\hline moyenne & 6 & \\
\hline bonne & 4 & \\
\hline $\begin{array}{l}\text { 7. Pensez-vous qu'il soit nécessaire de faire des essais cliniques en } \\
\text { médecine libérale? }\end{array}$ & 23 & 3 \\
\hline \multicolumn{3}{|l|}{$\begin{array}{l}\text { 8. Difficultés rencontrées (par ordre décroissant d'importance sur une } \\
\text { échelle de } 0 \text { à } 10 \text { (moyenne des réponses): }\end{array}$} \\
\hline manque de temps & 8,1 & \\
\hline méthodologie & 6,0 & \\
\hline argent & 3,6 & \\
\hline laboratoire & 4,4 & \\
\hline patient & 5,6 & \\
\hline entourage patient & 3,9 & \\
\hline autres & 6,0 & \\
\hline 9. Désirez-vous plus d'information sur les essais cliniques ? & 13 & 13 \\
\hline \multirow[t]{5}{*}{ 10. Moyens d'information préférés (par ordre décroissant) : } & 1. Internet & \\
\hline & 2. EPU soir & \\
\hline & 3. Formation sur $2 \mathrm{j}$ & \\
\hline & 4. DIU & \\
\hline & 5. Journaux médicaux & \\
\hline 11. Désirez-vous être un relais pour l'information de vos patients? & 17 & 9 \\
\hline \multicolumn{3}{|c|}{$\begin{array}{l}\text { a Document complémentaire avec commentaires medécins et analyse. Avec autorisation. Enquête réalisée auprès d'un groupement de médecins } \\
\text { investigateurs; Giacomino A, données non publiées. }\end{array}$} \\
\hline \multicolumn{3}{|c|}{ DIU = diplôme inter-universitaire ; EPU = enseignement post-universitaire ; FMC = formation médicale continue. } \\
\hline
\end{tabular}

nécessaires (89\% des investigateurs libéraux) [enquête réalisée auprès d'un groupement de médecins investigateurs ; Giacomino A, données non publiées].

2. La motivation pour les essais cliniques est variable selon les situations : $12 \%$ seulement des parents d'enfants accepteraient que leur enfant participe à un essai clinique, ${ }^{[10]} 72 \%$ de la clientèle de médecins investigateurs (enquête réalisée auprès de la patientèle de médecins investigateurs ; Giacomino A, données non publiées) se déclarent plutôt favorables à participer (13\% ont de fait déjà participé à des essais) ${ }^{[9]}$ et $25 \%$ des adultes toutvenant participeraient (taux de participation effective de $4 \%$ ). Autrement dit, en l'absence d'information de proximité, telle qu'une personne de l'entourage ou un médecin traitant lui-même impliqué dans les essais, le niveau de motivation est faible.

3. La connaissance du cadre des essais cliniques par les infirmières est très insuffisante (enquête réalisée au CHU de Tours ; Autret-Leca E, données non publiées). Si $90 \%$ des infirmières travaillant dans un $\mathrm{CHU}$, donc au contact des médecins investigateurs et des patients inclus dans des essais cliniques, savent qu'il existe une législation en matière d'essais cliniques, $45 \%$ ignorent que les protocoles d'étude sont soumis à un $\mathrm{CCPPRB},{ }^{[11]}$ et $36 \%$ seulement savent que l'avis du Comité est préalable à la conduite de l'essai (enquête réalisée au CHU de Tours ; Autret-Leca E, données non publiées).

4. Les patients de la clientèle d'investigateurs sont $19 \%$ seulement à considérer que l'information en matière d'essais cliniques est bonne et $86 \%$ souhaitent plus d'information (enquête réalisée auprès de la patientèle de médecins investigateurs ; Giacomino A, données non publiées).

5. Il existerait une suspicion sur l'information liée aux essais, particulièrement si celle-ci émane d'une source unique, a fortiori industrielle (enquête d'opinion ; Padioleau MF, données non publiées). 
6. Préférence est donnée à l'information de proximité (médecin traitant, presse) [enquête réalisée auprès de la patientèle de médecins investigateurs ; Giacomino A, données non publiées].

7. L'intelligibilité est une condition nécessaire. Un « traducteur » s'imposerait souvent.

8. La motivation des investigateurs (enquête réalisée auprès d'un groupement de médecins investigateurs ; Giacomino A, données non publiées) est liée au temps nécessaire à l'essai et à sa méthodologie.

9. Cinquante pour cent des investigateurs souhaiteraient être mieux formés/informés en matière d'essai, les moyens préférés étant Internet (auto-formation), les enseignements post-universitaires (EPU), les formations sur 2 jours consécutifs.

\section{Messages et cibles}

Deux types de messages peuvent être considérés. Ceux portant sur la recherche clinique en général et ceux dédiés à un essai en particulier.

\section{1 Information sur « LES » essais en général}

Les cibles sont le grand public, la presse générale comme spécialisée, les médecins investigateurs ou non, les autres professionnels de santé (pharmaciens, infirmiers, autres cadres de santé publics ou privés), les étudiants, les écoliers.

Les membres du groupe (institutionnels, industriels, consommateurs, patients, presse) sont parvenus à un accord sur les recommandations suivantes :

- Faire une formation ou délivrer une information sur une base régulière, et non en situation critique et donc émotionnelle comme c'est souvent le cas qui justifie les gros titres de la presse grand public.

- Développer la notion de citoyen «partenaire » et insister sur l'altruisme nécessaire pour que la recherche progresse même si l'intérêt direct du sujet participant n'est pas toujours évident.

- Faire comprendre que les essais sont « nécessaires » et que leurs résultats contribuent à l'amélioration de la Santé, dans son acception la plus large, qu'elle contribue par exemple à

Tableau III. Contrôle de connaissances réalisé dans le $\mathrm{CHU}$ de Tours auprès de 19 infirmières du CHU de Tours et de 51 médecins libéraux de la région Centre $^{a}$

\begin{tabular}{|c|c|c|}
\hline Question & Infirmières (\%) & Médecins libéraux (\%) \\
\hline \multicolumn{3}{|l|}{ 1. Existe-t-il une législation sur la recherche biomédicale en France? } \\
\hline oui & 90,0 & 90,0 \\
\hline non & 0,0 & 2,0 \\
\hline ne sait pas & 10,0 & 8,0 \\
\hline \multicolumn{3}{|l|}{ 2. Un médecin peut-il faire participer un patient à son insu? } \\
\hline oui & 5,0 & 4,0 \\
\hline non & 95,0 & 90,0 \\
\hline ne sait pas & 0,0 & 6,0 \\
\hline \multicolumn{3}{|l|}{ 3. Le CCPPRB : } \\
\hline est un comité d'éthique pour répondre aux patients pendant l'étude (oui) & 31,0 & 20,0 \\
\hline est un comité statuant sur la protection des personnes avant le début de l'étude (oui) & 36,0 & 60,0 \\
\hline ne sait pas & 32,0 & 20,0 \\
\hline \multicolumn{3}{|l|}{ 4. L'autorisation d'un seul parent suffit-il pour inclure un enfant dans un essai ? } \\
\hline oui & 11,0 & 8,0 \\
\hline non & 79,0 & 55,0 \\
\hline ne sait pas & 0,0 & 37,0 \\
\hline \multicolumn{3}{|l|}{ 5. Toute recherche biomédicale est déclarée aux autorités (DGS, Afssaps) : } \\
\hline oui & 74,0 & 61,0 \\
\hline non & 5,0 & 14,0 \\
\hline ne sait pas & 21,0 & 25,0 \\
\hline \multicolumn{3}{|l|}{ 6. Avant d'être mise sur le marché une molécule est obligatoirement testée sur: } \\
\hline animal uniquement & 0,0 & 0,0 \\
\hline animal et homme & 100,0 & 100,0 \\
\hline homme uniquement & 0,0 & 0,0 \\
\hline ne sait pas & 0,0 & 0,0 \\
\hline
\end{tabular}

a Enquête réalisée au CHU de Tours ; Autret-Leca E, données non publiées. Avec autorisation.

Afssaps = Agence française de sécurité sanitaire des produits de santé $;$ CCPPRB = Comité Consultatif de Protection des Personnes dans la Recherche Biomédicale ; DGS = Direction Générale de la Santé. 
l'évolution des pratiques médicales, à l'élaboration de recommandations de santé publique et de prise en charge thérapeutique des patients par leurs médecins traitants, à la formation des étudiants.

\subsection{Information sur « UN » essai en particulier}

Les cibles en sont l'investigateur, le médecin traitant, le patient et si besoin son entourage. Les recommandations :

- Le patient doit être traité en individu responsable, acteur de sa santé et libre de ses choix.

- Il faut se soucier de la prise en charge globale du sujet ayant accepté de participer à un essai clinique, autrement dit depuis son « accueil » initial dans la structure qui le recrute, puis pendant tout son suivi, et jusqu'au rendu des résultats. Cette absence de continuité et de respect de l'individualité dans la prise en charge explique sans doute des refus, et empêchera la «publicité » positive que le sujet pourra faire de l'essai (et au-delà de la recherche clinique) auprès de son entourage.

- L'information doit concerner toute la chaîne des soignants qui prennent en charge ce patient, pas son seul interlocuteur chargé de l'investigation clinique. Il convient de ne pas oublier que le médecin traitant habituel n'est qu'assez exceptionnellement l'investigateur, même s'il est informé et consent à la recherche pour son patient. Le « vécu » de ce médecin et d'autres soignants peut être déterminant sur sa confiance et sa volonté de voir son patient «partagé » entre activités de soins et de recherche, et donc entre médecin investigateur et médecin traitant.

\section{Informer de quoi ?}

\subsection{Pour améliorer le recrutement}

Deux expériences complémentaires ont été rapportées.

\subsubsection{L'expérience dite " $3 M$ "}

Cette expérience a consisté à développer une plate-forme d'information (numéro d'un centre d'appel, site internet dédié à l'essai) à destination du public sur un essai en cours dans l'herpès génital. L'essai multicentrique et multinational en ambulatoire présentait une difficulté de recrutement, particulièrement sur le territoire français. La plate-forme comprenait une publicité presse (figure 1).

Le développement de cette expérience a permis à son promoteur de prendre l'avis des différentes instances tutélaires (avis résumé plus haut dans la section réglementaire) mais surtout de dégager quelques pré-requis permettant d'éviter toute confusion

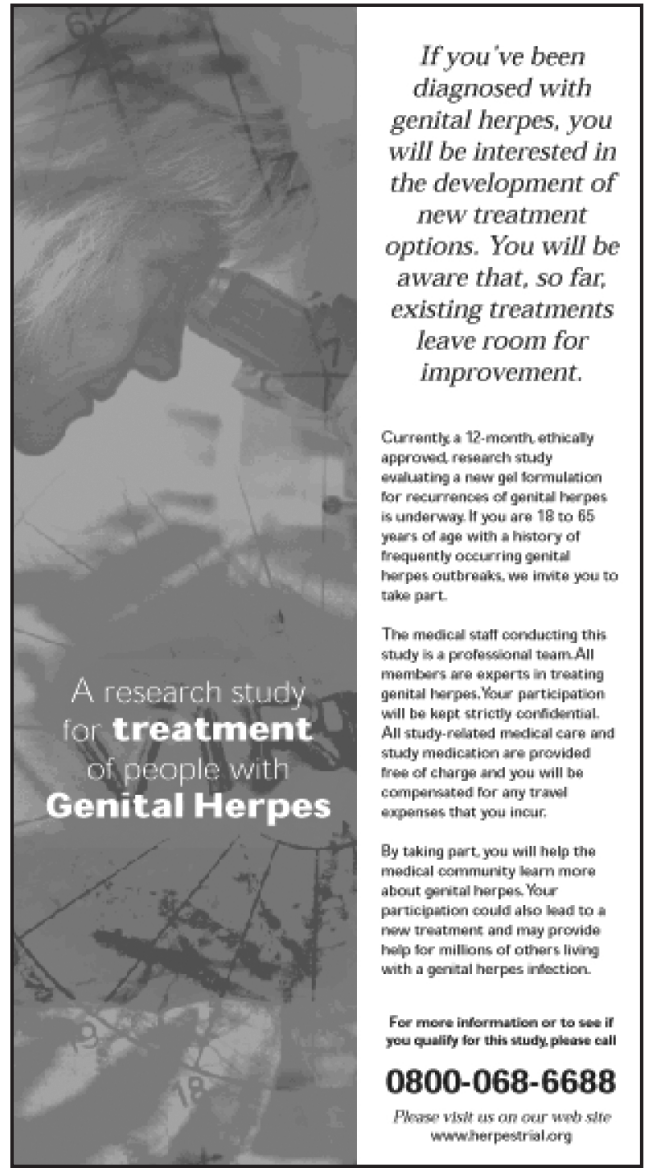

Fig. 1. Campagne de recrutement par voie de presse (Laboratoire 3M, avec autorisation).

entre recherche et publicité pharmaceutique. Ainsi seraient à éviter la mention du nom du traitement à l'étude, celle du nom du promoteur (discutable pour certains) et de l'identité du patient. En revanche, il faudrait faire apparaître intelligiblement la pathologie concernée, les caractéristiques de l'essai clinique et le mécanisme du traitement à l'essai.

Cette expérience, réitérée lors d'un essai suivant, s'est concrètement traduite par un gain de temps de recrutement (3 mois sur l'essai), et également sur la date de dépôt du dossier d'autorisation de mise sur le marché (AMM) [3 mois aussi] au prix toutefois d'un coût par patient considérable, potentiellement compensé par un gain en durée d'exploitation au plan commercial ultérieur.

\subsubsection{L'approche du CIC de Nancy}

L'option retenue a été une série d'annonces dans la presse locale décrivant la pathologie et les caractéristiques des patients recherchés. Ces derniers étaient invités à se mettre en rapport 
avec les infirmières du CIC, bien informées de l'essai, qui réalisaient le «centre d'appel » pour l'essai. Le résultat a été un recrutement considérablement facilité, et le coût (annonce, personnel) modique.

La question reste posée de la possible extension de ces expériences à l'ensemble des essais.

La discussion a ainsi retenu quatre points, globalement en faveur de la généralisation de l'expérience, qui sont : autant de propositions :

- Les promoteurs seraient, en Europe, en France particulièrement, plus inhibés par des préjugés d'ordre réglementaire que réellement contraints par un tel cadre. Il faut donc « oser » sans craindre, et entreprendre plus largement ce type de démarche, en en mesurant l'intérêt au cas par cas.

- Toute modalité de recrutement expose à des biais, celui de «professionnaliser» certains patients ou d'obtenir une sur-représentation de sous-populations particulières. Il faut donc y veiller au cas par cas.

- L'utilisation d'un « centre d'appel » semble être une bonne pratique, professionnalisant l'accueil et protégeant le promoteur et le patient.

- Les associations de patients peuvent être aussi un relais efficace et jouer ce même rôle, en toute indépendance du promoteur. Elles ne devraient cependant pas empêcher l'information en provenance directe du promoteur si celle-ci est souhaitée.

\subsection{Pour informer le patient à la fin de l'essai}

La loi du 4 mars $2002^{[4]}$ dispose que « ...la personne...EST informée des résultats GLOBAUX... ». Les CCPPRB recommandent désormais de faire figurer ce droit dans le formulaire d'information destiné au patient et accompagnant le recueil du consentement.

De la discussion sont ressorties les notions de retombées positives, mais aussi quelques difficultés.

Au plan des points positifs, la communication des résultats est un des éléments possibles de l'adhésion du patient, non tant à l'essai achevé qu'à des essais suivants, la possibilité d'un effet d'essaimage (« halo ») vis-à-vis d'autres patients potentiels. Cette obligation vis-à-vis du patient rappellerait en outre au promoteur de ne pas omettre d'informer tous les investigateurs des résultats globaux de l'essai.

Parmi les difficultés, on a pu relever le cas des essais négatifs (particulièrement où le placebo a été plus efficace ou mieux toléré que le produit à l'essai), avec la question posée d'insister ou non sur la «perte de chance » pour les patients inclus, les essais très longs, où une durée considérable peut séparer la sortie d'un patient de l'étude de la fin, et a fortiori de l'analyse de celle-ci. Enfin, les problèmes de confidentialité pour le promoteur de l'essai, non tant dans un souci de propriété intellectuelle ou concurrentielle que pour les raisons d'embargo liées à la communication et à la publication dans des médias internationaux aux règles strictes.

Suggestion : mettre à disposition cette information dès que disponible sur un site accessible au patient (voir 8.2 et tableau IV).

\section{Vecteurs de l'information}

\section{1 Les professionnels de santé}

Les professionnels de santé sont au premier rang des supports de la communication sur les essais.

Il s'agit des médecins investigateurs, mais aussi des médecins traitants, ce qui suppose leur formation et leur motivation à ce domaine. Une formation médicale initiale à la recherche clinique incluant la lecture critique d'articles ainsi qu' une formation continue en recherche clinique dans le cadre conventionnel (Caisse Nationale d'Assurance Maladie [CNAM]) ${ }^{[12]}$ sont des éléments indispensables à la participation des médecins traitants à l'information de leur patient. L'information par ce vecteur concerne toutes les étapes de l'essai, le patient et son entourage, le médecin traitant s'il n'est pas lui-même l'investigateur. L'Article 59-61 de la loi du 4 mars $2002^{[4]}$ fait obligation aux médecins de se former. Si la pratique du médecin s'étend aux essais cliniques, l'application directe devrait

Tableau IV. Sites concernant les essais cliniques

1. Cancer.gov : http://www.cancer.gov/

2. CenterWatch.com : http://www.centerwatch.com/

3. Search the studies : http://clinicalstudies.info.nih.gov/

4. ClincalTrials.gov : http://clinicaltrials.gov

5. Clinical Studies : http://www.nei.nih.gov/neitrials/index.htm

6. TrialsCentral : http://www.trialscentral.org

7. Current Controlled Trials : http://www.controlled-trials.com/

8. Actis : http://actis.org/index.html

9. ClinicalTrials.com : http://www.clinicaltrials.com/trials/trials.asp

10. Réseau canadien sur les essais cliniques :

http://www.hivnet.ucb.ca/list1fFrame.html

11. Essais européens : http://www.curatrials.com/

12. FNCLCC : http://www.fnclcc.fr/-sci/bect/clcc/clcc.htm

13. Arcat SIDA : http://www.arcat-sida.org/essais/content.html

14. INSERM : http://birbpi.inserm.fr:8001

http://onyx.tolbiac.inserm.fr/ (puis consultation/protocoles)

15. Afssaps : http://afssaps.sante.fr/htm/5/repec/repec0.htm 
être d'inclure la recherche clinique dans le champ de cette obligation.

Le pharmacien d'officine est un relais important de cette information, étant souvent le premier et le dernier relais d'information auprès du patient. Malheureusement, le pharmacien est probablement de tous les acteurs de la chaîne celui qui a le moins d'information sur le déroulement des essais, et ignore même auprès de qui se renseigner au cas où des patients les interrogeraient. L'information des pharmaciens permettrait au moins qu'ils ne soient pas un frein dans la réalisation des essais.

\subsection{Les répertoires}

Très développés aux Etats-Unis, limités à quelques initiatives en France, ils peuvent procurer une information générale sur les essais cliniques ou plus spécifiquement sur ceux qui s'appliquent à une pathologie (cancer, SIDA, maladies rares) et, pour un essai donné, remplir le double rôle d'informer sur son existence, son statut, les conditions pour y participer, les investigateurs ou les centres qui peuvent être contactés, et parfois délivrer une information générale sur les résultats si l'étude est terminée. Ils peuvent émaner de centres universitaires, de sites institutionnels ou gouvernementaux, de sociétés spécialisées dans le recrutement.

On trouvera dans le tableau IV une liste non exhaustive des sites les mieux connus, avec leur adresse.

Parmi les sites qui procurent - entre autres - une information générale sur les essais cliniques, cherchant à répondre à la question «qu'est-ce qu'un essai clinique ? », on peut citer le «Réseau Canadien de la Santé », le «Clinical Trial Program » de l'Université d'Indiana, «Clinical Trial Information for Patients », «Centerwatch » et «ClinicalTrials.gov ». Ce dernier, sans doute le plus célèbre, répertorie plus de 6000 essais conduits par des instances publiques et privées de par le monde. Parmi les sites orientés sur une pathologie, «Cancer.gov » présente plus de 1700 essais avec une version patients et une version professionnels de santé, et en France, différents sites universitaires, ou d'associations de patients (Cancer, SIDA). Certains sites ont plutôt comme vocation d'être des répertoires d'essais, s'adressant en priorité aux professionnels de santé, tels que «Current Controlled Trials », support du Cochrane Centre et du Medical Research Council en Grande-Bretagne avec un record de plus de 14000 essais enregistrés et classés, ainsi que le site où l'INSERM met en ligne une banque d'informations sur une centaine d'essais cliniques et épidémiologiques dans les hépatites B et $\mathrm{C}$. Mais on notera surtout la toute récente initiative de l'Afssaps qui vient de mettre sur son site internet (afssaps.sante.fr) un répertoire des essais cliniques qui, dans un premier temps, concerne les essais menés sur le traitement des maladies rares et des hépatites (figure 2).

Néanmoins, si cette approche se développe, elle se heurte encore à des obstacles de refus de certains promoteurs de voir leurs projets d'étude, et a fortiori leurs résultats, mis à la disposition d'un large public. Des réflexions et des initiatives tentent aujourd'hui de surmonter ces difficultés.

\subsection{Support aux investigateurs}

Parmi les éléments qui peuvent être mis à la disposition des investigateurs pour les aider dans leur mission d'information, citons des brochures concernant la recherche clinique en général ou thématique (ex : diabète), des affiches pour la salle d'attente (CIC de Nancy ${ }^{[13]}$ ).

En plus de la formation initiale et continue décrite plus haut, des réunions à thème permettraient $\mathrm{d}$ 'approfondir la relation médecin-malade en situation de recherche, et l'apprentissage du « switch » entre cette situation et celle de soins (jeux de rôles, par exemple). Des EPU plus sophistiqués seraient nécessaires pour la compréhension de certaines méthodologies qu'il s'agit parfois de simplifier pour être ensuite expliquées au patient, concernant par exemple les études de morbimortalité, qui posent des problèmes évidents pour être communiquées.

Un des supports importants, qui ne doit pas être négligé par le promoteur, est l'information en cours d'essai sur l'avancement de l'étude, les problèmes rencontrés de tous ordres par les autres investigateurs et leur solution, et l'information en fin d'essai. Cette dernière est elle-même reliée à l'obligation légale faite par la loi du 4 mars 2002. ${ }^{[4]}$ Un entraînement à délivrer cette information sensible aux patients et dans certains cas à leur famille est sans doute à prévoir également. Les centres d'appels eux-mêmes peuvent étendre la gamme de leurs services et être des supports utiles aux investigateurs (tableau V).

\subsection{Les médias}

Les actions vis-à-vis des médias seraient non seulement de les informer, afin qu'à leur tour ils disséminent cette information dans leurs colonnes, mais de les «former », c'est-à-dire de leur apporter les éléments leur permettant de saisir toute la problématique des essais et démystifiant certains éléments (rémunération des volontaires sains, honoraires des investigateurs, tirage au sort des sujets, choix de certaines populations d'essais, etc.). Il a été suggéré que l'Association des Journalistes Médicaux (AJMED) qui regroupe quelque 75 journalistes de la presse médicale spécialisée soit la plate-forme de cette expérience. 


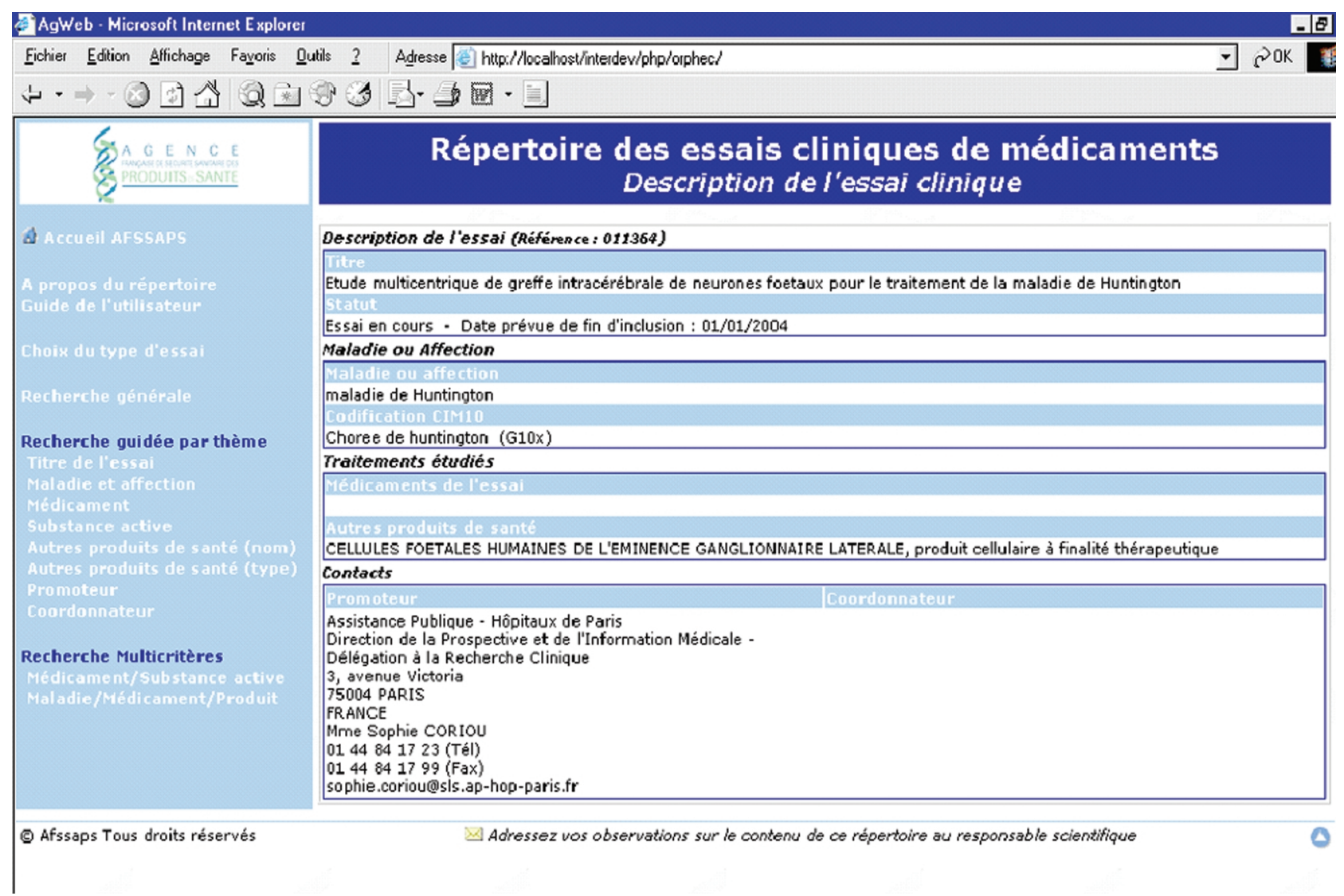

Fig. 2. Site Internet de l'Afssaps (Agence française de sécurité sanitaire des produits de santé) : répertoire des essais cliniques.

Aujourd'hui le constat est en effet que les médias bénéficient d'une information à deux vitesses, selon qu'ils appartiennent à des journalistes de la presse médicale ou à la presse grand public. Cette dernière voit son information toujours circonscrite au minimum avec des dossiers de presse peu consistants. Il en ressort que les journalistes se tournent vers leurs contacts personnels afin de « décoder » l'information utile, avec les aléas que ces choix comportent. Il sera difficile d'échapper totalement à la mise en exergue, parfois tapageuse, des chefs de rubrique, effectuant un travail proprement commercial à partir de celui, plus documenté, du journaliste. Il en reste qu'informer «plus vrai », c'est-à-dire pas seulement sur les «belles histoires » de la recherche clinique, mais aussi sur son quotidien, ses difficultés et ses échecs pourrait changer le regard du public. De même privilégier les aspects «pratiques » qui vont intéresser in fine le public des lecteurs (comment participer, comment se déroule un essai, qu'advient-il des soins pour l'affection en cours, où trouver l'information, comment mon médecin saura-t-il que je participe, aura-t-il son mot à dire, à quoi est-ce que je m'engage et quels sont mes droits, etc.) aiderait à la participation aux essais.

\section{Discussion et conclusions}

La plupart des éléments de discussion ont été soulevés lors de la table ronde et certains de ces points ont déjà été brièvement évoqués. Certains concernent des questions de fond, juridiques et éthiques, d'autres concernent des aspects pratiques et interrogent l'efficacité des campagnes de recrutement.

Parmi les questions légales, force est de constater l'inconfort des participants, malgré leur expertise, face à la question de la licité de recourir à des campagnes de recrutement. Les instances institutionnelles ont du mal à positionner leur avis et leur rôle dans ce cadre. Il est possible que la transposition en droit français de la Directive Européenne sur les Essais Cliniques ${ }^{[6]}$ permette de répondre à certaines de ces questions. Un point central à nos débats a été le recours à un «centre d'appel » dont l'indépendance 
Tableau V. Services offerts par un centre privé d'appels et d'assistance logistique pour les études (modifié, d'après Pro-Clinica data [Centre de Contacts et d'Assistance Logistique Médicalisé pour les Études $\left.{ }^{[14]}\right]$ )

\begin{tabular}{ll}
\hline Supports & Services \\
\hline Permanence téléphonique & Transmission d'informations sur les \\
& essais cliniques : multilinguistique \\
Service vocal interactif & Avis d'inclusion : randomisation \\
Internet & Questionnaire téléphonique de \\
& présélection \\
& Gestion des rendez-vous : relances \\
& Envoi de traitements, matériel, \\
& prélèvements \\
& Aide au remplissage de données : \\
& auto-journal (patient) ; CRF (médecin) \\
& Déclenchement d'interventions \\
& Gestion de tableaux de suivi \\
\hline CRF = case report form (cahier d'observations)
\end{tabular}

CRF $=$ case report form (cahier d'observations).

réelle, si tant est qu'elle soit recherchée, est discutable, et dont le fonctionnement impose de se pencher sur la question du secret médical. Le Code de Déontologie dispose que le patient lui-même ne peut délier le médecin à qui il confie des informations médicales le concernant de son obligation de réserve vis-à-vis des tiers en dehors des exceptions prévues par la loi dans le cadre d'investigations judiciaires, et à l'exception récente de l'extension aux autres soignants dans le cadre de son traitement en application de la loi du 4 mars 2002 déjà citée. ${ }^{[4]}$ Cependant rien ne semble empêcher le patient, comme tout citoyen, de confier toute information le concernant à des tiers à condition qu'il le fasse de son plein gré, et qu'il bénéficie des protections de la Loi Informatique et Libertés ${ }^{[15,16]}$ dès lors que ces données pourront faire l'objet d'une exploitation informatique, ce qui est le cas ici, le plus souvent. Selon les modalités de ce recueil, il appartient donc à ce «call centre » de préciser ses droits à la personne qui contacte, et de demander les autorisations nécessaires. Pour certains juristes cependant, il serait prudent de «matérialiser » le consentement du patient au recueil de données le concernant en faisant confirmer cet accord par le renvoi d'un document écrit et signé, établissant entre autres que le contact a été établi par le patient (pas de démarchage possible). ${ }^{[14]}$ Le tableau $\mathrm{V}$ indique les services que peuvent offrir aujourd'hui les centres d'appels.

Parmi les questions de fond se pose également celle de comprendre la différence de pourcentage, quelle que soit l'enquête considérée, entre patients considérant l'intérêt de la recherche clinique, patients envisageant éventuellement d'y participer et participation effective. Entre les deux premières réponses, l'écart est faible et traduit le fond altruiste existant en chacun, une fois la situation expliquée. L'attrition se situe en fait autour de la participation. L'enquête réalisée à Saint-Etienne avait mis en évidence que la perception du risque dans la population générale jouait un rôle central (enquête réalisée auprès d'un groupement de médecins investigateurs ; Giacomino A, données non publiées). Rappelons que la révision en 2000 de la Déclaration d'Helsinki ${ }^{[17]}$ est allée jusqu'à poser indirectement la question de la licité de la recherche clinique, en s'opposant, dans sa première version, à la conduite de certains essais (notamment contre placebo dès lors qu'un traitement est disponible) sans lesquels toute réelle connaissance est impossible. La publicité faite autour de certaines affaires concernant le respect du droit à l'alternative thérapeutique dans certaines affections graves peut ajouter à ces perceptions. ${ }^{[18]}$ On doit admettre qu'il existe un paradoxe que la recherche clinique doit gérer : le fait même de conduire des essais cliniques chez l'homme ne traduit-il pas notre besoin de connaissance et donc notre ignorance relative des effets des traitements ? Et la recherche d'un risque le plus proche possible de zéro pour un médicament commercialisé pour une grande population n'implique-t-il pas en retour l'obligation d'accrôitre sans cesse le nombre de patients devant être «exposés » au stade de la recherche sur le médicament, et donc la probabilité d'observer des problèmes liés à la tolérance ? Les «megatrials » incluent aujourd'hui plus de 20000 patients pour tenter de dégager des bénéfices dont l'amplitude peut pour certains paraitre marginale. Ils sont pourtant le passage obligé de la connaissance pour qui veut mener une politique de santé éclairée. En même temps l'écho fait à tout propos au «Principe de Précaution » transforme celui-ci en principe d'abstention, c'est dire qu'il inquiète autant qu'il est censé rassurer. Par extension, l'ampleur des précautions réglementaires mises autour des essais, si elle peut rassurer certains, justifient d'autres dans leur perception de la dangerosité potentielle des essais. L'ambivalence joue donc un rôle plutôt inhibiteur. A contrario, l'explicitation du risque au patient qui s'apprête à participer à un essai clinique donné aboutit rarement à son refus, pas plus que la signature du consentement. Il s'agit donc plutôt d'une perception ambiante pour les sujets n'ayant pas encore participé à des essais. Une des missions que devrait s'assigner toute communication vers le grand public est de rassurer.

La confidentialité des données sensibles et personnelles, et plus récemment celle de la propriété et de l'accès à certaines bases de données, peut être un frein. La presse s'est largement fait l'écho de ces questions à propos des données du génome, et du phénotypage de populations entières. Le débat court sur la propriété et l'exploitation en matière génomique et protéomique. ${ }^{[19]}$ Bien que la portée individuelle en soit encore au stade virtuel dans la plupart des cas, l'idée plus générale que la recherche clinique pourrait somme toute dérober à son insu des informations touchant au sanctuaire génétique de l'individu inquiète. 
L'efficacité des campagnes de recrutement a été en toile de fond des discussions. Cependant les exemples présentés offraient des preuves individuelles d'efficacité. Le premier point est le rapport possible entre information générale sur les essais et participation à des essais. En d'autres termes, est-ce que lancer des campagnes de sensibilisation, former les médias et les professionnels changera in fine le taux de participation aux essais ? Et la question subsidiaire est évidemment : est-ce que cet effet, s'il est observé, se maintiendra dans le temps ? Un premier élément de réponse provient des enquêtes menées pour la table ronde. En effet, on constate qu'il existe un gradient d'intérêt de 1-3 selon la proximité ou la non-proximité du patient à une source d'information concernée (médecin traitant investigateur, entourage) : $25-75 \%$ pour l'information et $4-12 \%$ pour la participation. Ce gradient semble inférer également d'un lien entre information et participation.

Néanmoins les efforts déployés par l'Assistance Publique pour informer les patients sur les essais se traduisent dans une participation de $3 \%$ seulement, (Données non publiées, Assistance Publique - Hôpitaux de Paris) soit au mieux le taux observé spontanément. Il est possible que d'autres freins expliquent ces particularités. Parmi eux, la différence pouvant exister entre une information personnalisée et humanisée (le médecin, l'entourage) et une information désincarnée (livret, note, spot TV, annonce presse). Une enquête récente faite aux Etats-Unis ${ }^{[20]}$ montre que $61 \%$ des américains adultes font confiance à leur médecin en matière d'information médicale, $35 \%$ à une infirmière, $26 \%$ à Internet, $14 \%$ à la télévision, $13 \%$ et $12 \%$ respectivement aux magazines et aux journaux. Ces données recoupent les nôtres, à l'exception toutefois de la presse écrite, mieux placée que les autres médias, à condition de parler de presse régionale. On retrouve donc la notion de proximité. Concernant le maintien dans le temps, il est intéressant de se tourner vers les Etats-Unis où la pratique existe depuis plusieurs années. Selon une enquête récente ${ }^{[21]}$ concernant l'état d'esprit à propos des essais cliniques, le pourcentage de personnes qui disent être assurément ou probablement intéressées par participer à une recherche clinique a diminué de $35 \%$ à $31 \%$ entre 1998 et 2002. Dans le même temps et paradoxalement, la connaissance du public sur les essais, selon cette même enquête, a augmenté de $41 \%$ à $55 \%$. Quand on regarde enfin le taux de participation effective aux essais, il est resté stable à $4 \%$ pendant cette même période. La question est donc posée, d'une part du rapport pouvant ou non exister entre connaissance générale sur les essais et souhait de participation, et sur l'efficacité ultime en termes de recrutement, puisque celui-ci est faible et apparemment indifférent aux fluctuations d'opinion. Ceci amène à se poser la question de savoir s'il n'existe pas d'une part une connaissance globale et sans lien direct avec la motivation personnelle, et d'autre part une motivation directement mise en jeu dès que le patient est concerné ou sait qu'il peut l'être personnellement. Par conséquent, si l'on ne devrait pas réserver l'information générale pour rectifier des perceptions erronées et préparer, en le sensibilisant, le public à recevoir une information plus spécifique à un moment ou un autre et, à l'inverse, utiliser les campagnes pour le recrutement des personnes concernées, donc par les moyens de proximité les plus appropriés (tableau $\mathrm{V}$ ).

En forme de conclusion, la table ronde a finalement émis un certain nombre de recommandations résumées ici :

- ne pas créer de nouvelles contraintes réglementaires ;

- oser, pour les promoteurs, utiliser l'information directe du public ;

- élaborer une charte professionnelle du bon usage de l'information destinée au public ;

- impliquer dans la communication toute la chaîne des professionnels ;

- privilégier l'information de proximité (médecin traitant et presse régionale, en priorité) ;

- encourager le développement des sites et répertoires d'essais cliniques ;

- développer une vraie pédagogie pour les non-professionnels de l'essai clinique ;

- former les médias aux bases de la recherche clinique.

\section{Participants}

E. Autret-Leca (Hôpital Bretonneau, Tours), Ch. Belorgey (Afssaps, Saint-Denis), M. Bernard-Harlaut (CNAFAL, Paris), D. Cellier (MerckLipha, Lyon), F. Chazelle (MSD, Paris), M.F. Chevallier-Le Guyader (INSERM, Paris), M. Couderc (Sanofi-Synthélabo, Le Plessis-Robinson), D. Debiais (AMGEN, Neuilly-sur-Seine), J. D'Enfert (Aventis, Paris), A.M. Duguet (CCPPRB Toulouse 2, Toulouse), L. Fleury (Afssaps, Saint-Denis), M. Gallard (LEEM, Paris), A. Giacomino (MG France, Avoine), Y. Juillet (LEEM, Paris), H. Kolsky (3M Santé, Cergy-Pontoise), J.P. Lehner (SanofiSynthélabo, Le Plessis-Robinson), F. Lemaire (CHU Mondor, Créteil), M.F. Padioleau (AJMED, Paris), M. Peschanski (INSERM U421, Créteil), S. Ravoire (Lilly France, Saint-Cloud), J.P. Reynier (Faculté de Pharmacie, Marseille), Ph. Ricordeau (CNAMTS, Paris), P. Sassano (Novartis, RueilMalmaison), C. Sibenaler (LEEM, Paris), M.C. Simon (ANRS, Paris), J. Soletti (LEEM, Paris).

\section{Références}

1. Ca m'intéresse. 2002 0ctobre : n²60

2. Zannad F, Plétan Y, et les participants de la table-ronde $n^{\circ} 2$ de Giens. Difficultés à la réalisation des essais cliniques en France. Thérapie 2000; 56: 341-7

3. Le Public et les Essais Cliniques. 10ème Journée de l'Association pour le Développement de la Pharmacologie Clinique, Paris, 22/10/1997. Lettre Pharmacologue 1998; 12 (5): 91-101

4. Loi relative aux droits des malades et à la qualité du système de soins adoptée le 4 mars 2002. Bulletin Officiel de la République Française du 5 mars 2002 : article 59-61 
5. Loi portant sur la transposition de deux directives européennes relatives à la publicité pour les médicaments à usage humain, aux médicaments homéopathiques à usage humain, aux dispositifs médicaux, à l'exercice de la pharmacie et à la prévention du tabagisme. Bulletin Officiel de la République Française : Loi n ${ }^{\circ}$ 94-43 du 18 janvier 1994

6. Directive 2001/20/CE du Parlement européen et du Conseil du 4 avril 2001 concernant le rapprochement des dispositions législatives, réglementaires et administratives des États membres relatives à l'application de bonnes pratiques cliniques dans la conduite d'essais cliniques de médicaments à usage humain [online]. Journal Officiel des Communités Européennes nº121-1/05/2001. Available from URL: http://europa.eu.int/eur-lex/fr/archive/2001/ 1_12120010501fr.html

7. US Food and Drug Administration. FDA Modernization Act, 1997: section 113 [online]. Available from URL: http://www.fda.gov/opacom/7modact.html [Accessed 2003 Aug 25]

8. Pharmaceutical Research and Manufacturers of America (PhRMA). Principles on conduct of clinical trials and communication of clinical trial results. 2002, 8-e

9. Jaillon P, Detchevery R, Lacondemine H, et al. L'étude d'opinion 10 ans après : 1987-1997. Lettre Pharmacologue 1998; 12 (5): 92-6

10. Autret E, Dutertre JP, Barbier P, et al. Parental opinion about biomedical research in children. Dev Pharmacol Ther 1993; 20: 64-71

11. Benhamou-Jantelet G. Nurses ethical perceptions of health care and medical research: an audit in a French hospital. Nursing Ethics 2001; 8: 2002

12. Loi portant création création de la couverture médicale universelle (CMU). Loi $\mathrm{n}^{\circ}$ 99-641 du 27 juillet 1999 : article 56
13. Centre d' Investigation Clinique de NANCY (Nancy CIC) [online]. Available from URL: http://www.chu.nancy.fr/CIC

14. Fournie X. Avantages et conditions d'utilisation du contact direct avec les patients dans l'évaluation de la santé. Pro-Clinica (Centre de Contacts et d'Assistance Logistique Médicalisé pour les Études), 2002. (Data on file)

15. Loi Informatique et Libertés : loi relative aux fichiers et aux libertés. Loi ${ }^{\circ}$ 78-17 du 6 janvier 1978

16. Loi Informatique et Libertés : loi relative au traitement des données nominatives ayant pour fin la recherche dans le domaine de la santé. Loi n ${ }^{\circ} 94-548$ du 1 juillet 1994

17. Déclaration d'Helsinki : Recommandations à l'adresse des Médecins dans le domaine de la recherche biomédicale portant sur des sujets humains. $52^{2 \mathrm{eme}}$ Assemblée générale. Octobre 2000 ; Edinburgh

18. Chambre Correctionnelle de Paris. Citation dans Le Monde du 15/09/02. Le Monde 2002 Sept 15

19. Wall Street Journal 2002 Oct 24

20. Gashin A, Ward S. USA Today 2002 Oct 23

21. Appl Clin Trials 2002: 8 Aug

Correspondance et offprints : Fä̈ez Zannad, CIC-INSERM-CHU de Nancy, Hôpital Jeanne d'Arc, 54201 Dommartin les Toul Cedex, France.

E-mail : cic@chu-nancy.fr 